\title{
UNA METODOLOGÍA PARA EL DISEÑO DE UN CURRÍCULO ORIENTADO A LAS COMPETENCIAS
}

\section{A METHODOLOGY FOR COMPETENCY ORIENTED CURRICULA DESIGN}

\author{
Rodolfo Schmal S. ${ }^{1} \quad$ Andrés Ruiz-Tagle A. ${ }^{2}$ \\ Recibido el 9 de julio de 2007, aceptado el 19 de noviembre de 2007 \\ Received: July 9, 2007 Accepted: November 19, 2007
}

\begin{abstract}
RESUMEN
En este artículo se aborda el desafío del diseño de un currículo orientado al logro de competencias. Para ello, se analizó el estado del arte mediante una revisión del concepto de competencia y de los modelos curriculares en este ámbito. De esta revisión no se ha encontrado un método formal que permita construir un currículo orientado a las competencias. Para cubrir esta brecha el presente trabajo propone una metodología cuyo punto de partida es asociar a cada competencia un módulo o una asignatura, asociación que puede ser modificada por restricciones de tiempo y recursos a través de un proceso de refinamientos sucesivos.

La metodología propone las siguientes etapas a partir de un perfil profesional específico dado por sus competencias y capacidades asociadas: a) identificación de los módulos; b) secuenciación de los módulos; c) estructuración de los módulos; d) revisión de los módulos; e) revisión del currículo, y f) construcción del syllabus.

Bajo esta metodología los módulos que conforman el currículo apuntan a integrar los conocimientos y las habilidades requeridas para el logro de las competencias, antes que a fraccionarlos por disciplina.
\end{abstract}

Palabras clave: Currículo, competencias, modelo curricular, metodologías, diseño curricular.

\section{ABSTRACT}

This article deals with the challenge of designing a competency-based curriculum. For this a revision of the concept of competence was analyzed along with the existing curricula models based on competence. A formal method wasn't found which would have allowed in constructing a competency-based curriculum. In order to solve, this article proposes the methodology with which a first stage is started associating each competence with one module or assignment which perhaps at a later stage could be modified for time and resource restrictions through successive refinement process.

Starting from a specific professional profile defined by its competences and associate capacities the methodology proposes: a) identification of modules; b) module sequencing; $c$ ) structuring of module; $d$ ) revision of each module; e) revision of curriculum; andf) construction of syllabus for each module.

It is through this methodology the modules that are part of the curriculum aims to integrate the knowledge and the skills required to achieve the competence.

Keywords: Curricula, competences, competency-based curriculum, methodology, curricula design.

\section{INTRODUCCIÓN}

En las últimas décadas se ha producido un significativo incremento en la cantidad de estudiantes universitarios. En las figuras 1 y 2 se puede observar la diferencia de cobertura entre dos generaciones de distintos países, aquella comprendida entre los 25 y 34 años y entre los 45 y 54 años [1]. Esta mayor cobertura está posibilitando el acceso a las universidades de sectores socioeconómicos de menores ingresos que en el pasado veían limitada su incorporación por no terminar su educación secundaria y/o por la necesidad de incorporarse tempranamente al mundo del trabajo. En ambas figuras el promedio OCDE (Organización para la Cooperación y Desarrollo Económico) corresponde al promedio de los países más desarrollados.

\footnotetext{
1 Facultad de Ciencias Empresariales. Universidad de Talca. 2 Norte 685. Casilla 721. Talca, Chile. E-mail: rschmal@utalca.cl

2 Facultad de Ciencias Empresariales. Universidad de Talca. 2 Norte 685. Casilla 721. Talca, Chile. E-mail: aruiz-tagle@utalca.cl
} 


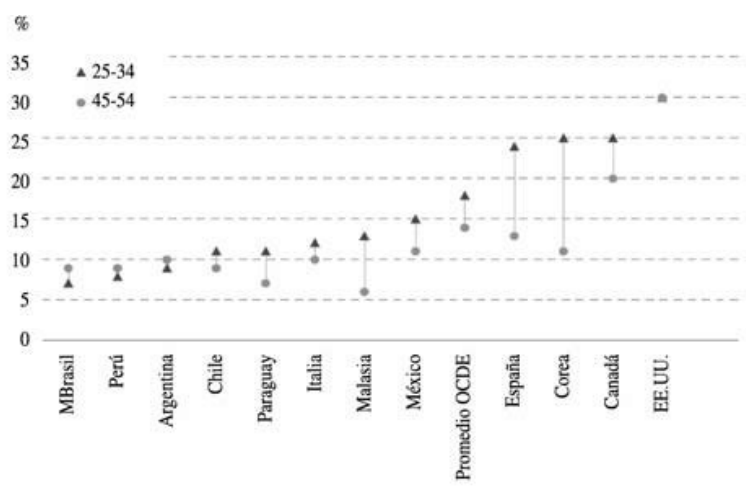

Figura 1. \% de población con al menos educación secundaria, según tramo de edad.

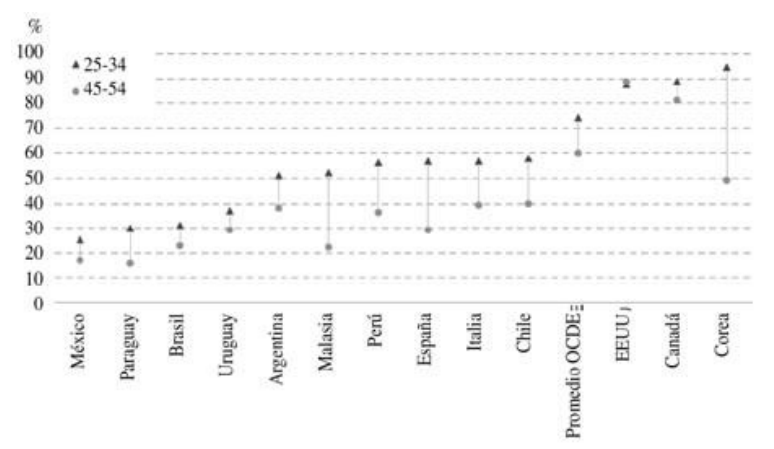

Figura 2. \% de población con educación terciaria tipo A, según ${ }^{3}$ tramo de edad. Fuente: MINEDUC, 2003.

En Chile, esta ampliación de la cobertura se ha dado bajo débiles marcos de regulación, tanto para la creación de nuevas instituciones de educación superior, como de nuevas carreras y/o la apertura de nuevas vacantes. Junto con ello, se ha planteado que la inserción de los países en el mundo actual está demandando cotas más altas de productividad y de igualdad de oportunidades en el acceso a la educación. Para las próximas décadas los cambios que se vislumbran en las universidades apuntan a superar problemas asociados a la ausencia de regulación -o de regulación por el mercado- que afectan a la calidad de la educación, los cuales están empujando al establecimiento de esquemas de acreditación y de información que orienten la asignación de recursos públicos, el financiamiento de la educación en general y a la vinculación de las universidades con el sector productivo.

\footnotetext{
3 La educación terciaria corresponde a la educación superior y los programas de educación terciaria tipo A son programas basados principalmente en teoría y diseñados para proveer conocimientos que permitan continuar en programas de investigación avanzada y profesiones con altos requerimientos de conocimientos.
}

La inserción de Chile en el mundo por la vía de un modelo de desarrollo basado en las exportaciones en un contexto de globalización y altamente competitivo, plantea profundos desafíos al sistema educativo en su conjunto, muy particularmente al sistema de educación superior y, en él, al sistema universitario. Retos en materia de formación de profesionales y de investigación y desarrollo que posibiliten encarar con éxito la necesidad de incrementar la productividad de la sociedad chilena.

La Universidad de Talca se ha propuesto abordar estos retos y desde el año 2004 ha realizado una transformación curricular de sus carreras de pregrado en el llamado Proyecto de Rediseño Curricular Basado en Competencias. En el marco de este proceso surgió la necesidad de desarrollar una metodología de trabajo que permitiera -a partir de un vector de competencias asociado a un perfil profesional específico- culminar en un currículo o plan de estudios orientado a la formación de las competencias consignadas en el vector correspondiente.

\section{ANTECEDENTES}

El enfoque basado en competencias surge en EEUU a comienzos del siglo pasado en el ámbito de la capacitación laboral y de las prácticas con miras a acercar a los estudiantes al mundo laboral real. Sin embargo, recién con los profundos cambios económicos y tecnológicos que se desatan desde los años 70 el concepto empieza a adquirir relevancia. Estos cambios plantean nuevos desafíos en educación y capacitación que respondan a las exigencias empresariales para incrementar su eficiencia, productividad y rentabilidad. En este contexto, las competencias de los recursos humanos disponibles pasan a ser un factor clave, no sólo para la consolidación y proyección de las empresas, sino para su supervivencia misma.

Desde entonces se busca una mayor vinculación entre las universidades y las empresas que disminuya la brecha dada por el énfasis que la docencia universitaria otorga a la adquisición de conocimientos, por sobre la aplicación de ellos en las empresas.

La educación basada en competencias es una forma de educación que deriva en un currículo a partir de un análisis prospectivo de la sociedad y del intento por certificar el progreso de los estudiantes sobre la base de un rendimiento o comportamiento demostrable en una o varias de las competencias exigidas [2].

La gran diferencia de este enfoque, con respecto al tradicional, es que la competencia no proviene solamente de la aprobación de un currículo basado en objetivos 
cognitivos, sino de la aplicación de conocimientos en ambientes reales, abriendo la posibilidad de transformar experiencias de aprendizajes en la posesión de competencias. Esto es, una educación orientada a la generación de competencias asume que el foco está puesto en los resultados del aprendizaje.

McClelland sostiene que el cambio de foco desde la evaluación de la inteligencia hacia la evaluación de la competencia está ayudando a un diseño curricular centrado en quien está aprendiendo, y que está basado en el pensamiento sistémico y la planificación estratégica [3]. No obstante, este diseño curricular basado en competencias enfrenta problemas en el ámbito de la medición del aprendizaje y de la implementación del cambio por las modificaciones que conlleva, particularmente de recursos docentes, metodológicos, de tiempo y de las redes con empresas.

A pesar de estas dificultades, Larraín y González afirman que existen poderosas razones para explicar el interés por implementar un enfoque orientado a las competencias en la formación de profesionales universitarios [4]:

- permite evaluar mejor los aprendizajes al momento de egreso;

- posibilita una expedita comunicación con los empleadores y proporciona mayores garantías de las capacidades de los egresados;

- facilita la inserción laboral y ajusta la oferta a los requerimientos para distintas áreas de desempeño profesional, $\mathrm{y}$

- genera mayor productividad temprana de los egresados.

La puesta en marcha de un currículo por competencias implica una serie de desafíos:

i) en el diseño curricular y la docencia: nuevo rol del docente, orientado al aprendizaje antes que a la enseñanza, mayor relación entre la teoría y la práctica, por lo que se requiere mayor vinculación con el sector productivo;

ii) en la gestión institucional: mejoramiento continuo, formación a lo largo de la vida, certificación de competencias, $y$

iii) en el sistema global: tránsito entre el aula y la práctica laboral, avances progresivos por módulos.

Si bien el interés por las competencias tiene su origen en la necesidad de impulsar una educación capaz de satisfacer los requerimientos de una realidad laboral cada vez más exigente, el desarrollo de este enfoque ha logrado ir más allá, incluyendo aspectos que no solo se relacionan con el mundo empresarial, sino con el ejercicio de la ciudadanía y el desarrollo de las personas, las que cobran especial importancia con el ingreso a las universidades de alumnos con bajo capital sociocultural.

Lo anterior se da en un contexto en el que las tecnologías de información y comunicación están alterando sustantivamente la manera como fluye la información al posibilitar que la comunicación fluya de "muchos a muchos" en vez de "uno a muchos". Las fuentes de aprendizaje ya no son monopolizadas por el profesor, sino que provienen de múltiples fuentes no tradicionales tales como los buscadores en Internet y la comunicación electrónica. De esta forma se ha abierto la posibilidad de que las tradicionales clases frontales centradas en el profesor que enseña (de experto a novato), sean sustituidas y/o complementadas por relaciones cuyo eje sea el alumno que aprende sobre la base de discusiones críticas en las cuales el "novato" también sea capaz de hacer aportes valiosos al "experto" [5].

\section{EL CONCEPTO DE COMPETENCIA}

En USA el Internacional Board of Standard for Training and Perfomance Instruction (IBSTPI) define una competencia como "un conocimiento, habilidad o actitud que habilita a una persona para desempeñar efectivamente las actividades asociadas a una ocupación o función de acuerdo a los estándares esperados en el empleo" [6]. Por su parte, el National Center for Education Statistics (NCES) define una competencia como "la combinación de conocimientos, habilidades y actitudes necesarias para realizar una tarea específica" [7].

Según Sladogna toda competencia es una síntesis de las experiencias que el sujeto ha logrado construir en el marco de su entorno vital amplio, pasado y presente. Por tanto, las competencias son capacidades complejas que poseen distintos grados de integración y se manifiestan en una gran variedad de situaciones en los diversos ámbitos de la vida humana personal y social [8].

Earnest y De Melo definen la competencia como una sentencia que describe la demostración integrada de un agrupamiento (cluster) de capacidades relacionadas y de actitudes observables y medibles en la ejecución de un trabajo o una tarea [9]. Esto implica que:

a) la competencia es medible en términos de cantidad, calidad, tiempo, costo o una combinación de ellos, cuya acción o rendimiento orienta los verbos a usar al describirla;

b) un cluster de capacidades consiste en capacidades cognitivas, prácticas y sociales manejadas diestramente como un todo; 
c) las capacidades envuelven tanto las capacidades cognitivas de alto nivel especificadas en la taxonomía de Bloom [10] -analizar, interpretar, diseñar, evaluar, crear, planificar, resolver, diagnosticar, etc.- como las capacidades prácticas de bajo nivel de la taxonomía de Dave [11] -cortar, juntar, medir, soldar, pintar, etc.-;

d) un trabajo es una actividad con un inicio y un fin definidos a ser realizado en un período de tiempo, independiente de otro trabajo, y que da como resultado un producto, servicio o decisión;

e) el desempeño de un trabajo debe serlo por sobre un nivel predeterminado en todo momento que se realice para que sea válido y confiable.

Siguiendo a Larraín y González [4], la competencia es concebida como "una concatenación de saberes, no sólo pragmáticos y orientados a la producción, sino aquellos que articulan una concepción del ser, del saber, saber hacer, del saber convivir". La competencia otorga "un significado de unidad e implica que los elementos del conocimiento tienen sentido sólo en función del conjunto. En efecto, aunque se pueden fragmentar sus componentes, éstos por separado no constituyen la competencia: ser competente implica el dominio de la totalidad de elementos y no sólo de alguna(s) de las partes". Por tanto, la competencia es "un saber en acción. Un saber cuyo sentido inmediato no es describir la realidad, sino modificarla; no definir problemas, sino solucionarlos; un saber el qué, pero también saber cómo". La competencia se concibe como una capacidad efectiva para llevar a cabo de manera exitosa una actividad plenamente identificada que se desarrolla a través de experiencias de aprendizaje en cuyo campo de conocimiento se integran 3 saberes: conceptual (saber conocer), procedimental (saber hacer) y actitudinal (saber ser). En relación a la competencia se pueden distinguir 3 enfoques: aquel que se centra en la capacidad de ejecutar las tareas; aquel que se concentra en los atributos personales (actitudes, capacidades), y el holístico, que incluye los dos anteriores.

El concepto de competencia más generalizado y aceptado es el de "saber hacer en un contexto". El "saber hacer", lejos de entenderse como "hacer" a secas, requiere de conocimiento (teórico, práctico o teórico-práctico), afectividad, compromiso, cooperación y cumplimiento, todo lo cual se expresa en el desempeño, también de tipo teórico, práctico o teórico-práctico. Por ejemplo, cuando alguien lee un texto y lo interpreta (saber hacer) ejecuta una acción (desempeño) en un contexto teórico (contenido del texto) $[4,5]$.
En consecuencia, el concepto de competencia integra conocimientos, potencialidades, habilidades, destrezas, prácticas y acciones de diversa índole (personales, colectivas, afectivas, sociales, culturales) en diferentes escenarios de aprendizaje y desempeño.

Desde el punto de vista de los actores involucrados, en las empresas la competencia es vista en términos de capacidades para realizar los trabajos que se llevan a cabo en ella; en cambio, el desarrollador de currículo y el educador piensan en términos de las capacidades prácticas, cognitivas y sociales que habrán de desarrollarse en los estudiantes.

Si bien el concepto proviene del mundo laboral y tiene una fuerte connotación utilitaria, en la actualidad esta concepción ha evolucionado de forma tal que una competencia envuelve la demostración de la capacidad para un desempeño satisfactorio no sólo en ámbitos definidos dentro de las organizaciones, sino que, más allá de ellas, en la sociedad en que nos desenvolvemos.

Por tanto, se puede afirmar que la competencia es la puesta en acción del conocimiento, lo que implica que un diseño curricular orientado a la formación de competencias debe estar guiado en lo sustancial por estrategias que destaquen la relevancia y pertinencia de los contenidos que se aprenden; eviten el fraccionamiento tradicional de los conocimientos y faciliten su integración; generen aprendizajes en situaciones complejas y, por último, estimulen, faciliten y provoquen la autonomía personal del estudiante.

\section{MODELOS DE CONSTRUCCIÓN CURRICULAR}

Se presentan dos modelos de diseño de una arquitectura curricular basada en competencias desarrollados uno en USA y el otro en India. El primero, cuyo proceso general se muestra en la figura 3 , es el que se lleva a cabo en el Instructional Perfomance Technology de la Boise State University para desarrollar su currículo basado en competencias [12]. Dentro de este proceso, las etapas 1, 2, 3 y 4 están destinadas a identificar las competencias, en tanto que la etapa 5 diseña los módulos, transformando las competencias en programas de estudio e implementación. En la etapa 6 se evalúa la efectividad del currículo resultante. 


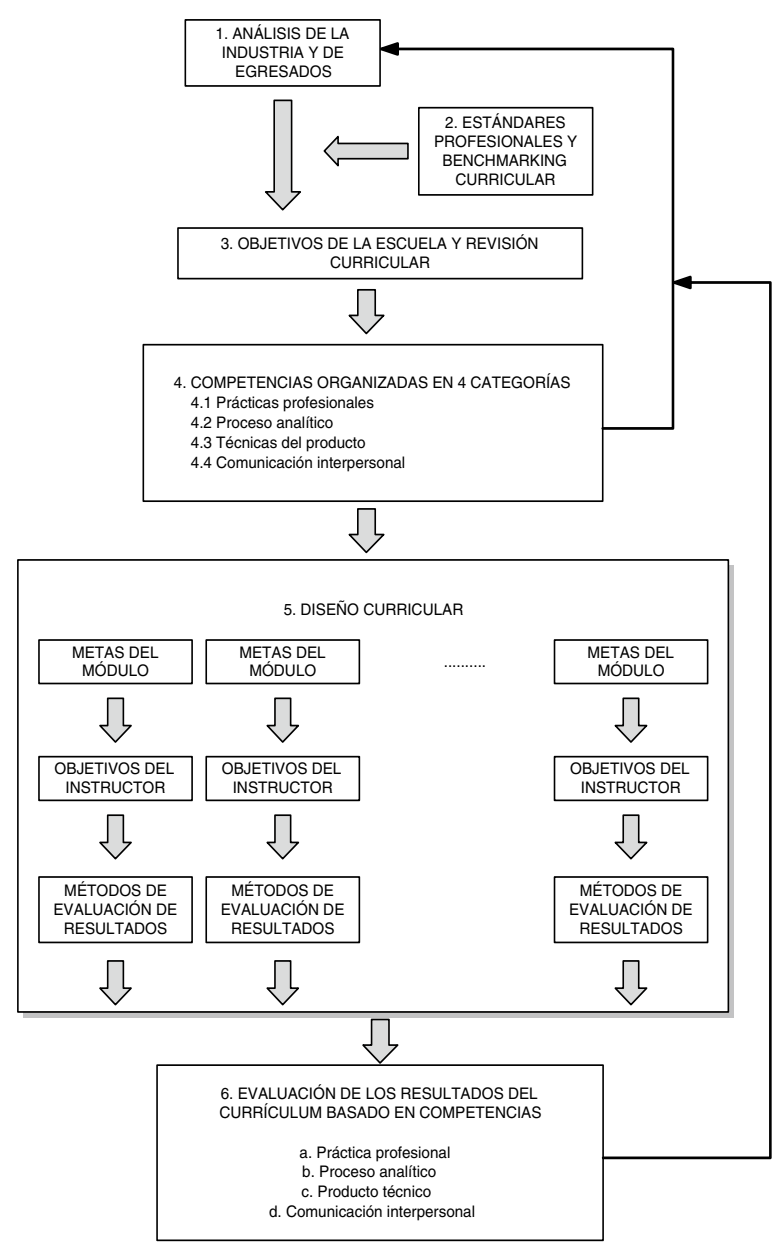

Figura 3. Modelo IPT (Instructional Perfomance Technology). Fuente: [12].

En la etapa 1 se aplica una encuesta a los graduados que debe realizarse anualmente en la universidad destinada a mostrar la mayor/menor efectividad en producir las competencias. Sus resultados son analizados por un comité de arquitectura curricular (CAC) conformados por académicos de las disciplinas comprometidas. Esto se explica por la alta interacción que en USA suelen tener los académicos con la realidad empresarial, realidad que no se da en otros países no obstante los esfuerzos que se realizan en esa dirección.

La etapa 2 involucra establecer los estándares profesionales y el benchmarking curricular. El CAC revisa las competencias declaradas por las organizaciones profesionales correspondientes y revisa el currículo de otras 4 universidades que ofrezcan programas académicos similares. Esta revisión permite al comité formular un rango de competencias que los estudiantes deben poseer una vez graduados.
La etapa 3 está destinada a identificar las metas de la escuela por parte del CAC, las que son comparadas con las competencias identificadas en la etapa 2 para los efectos de agregar competencias que puedan ser consideradas importantes, así como modificar y/o eliminar otras.

En la etapa 4 el CAC sintetiza las capacidades, competencias y metas identificadas en las etapas anteriores describiendo las competencias que los estudiantes deben poseer una vez graduados. El resultado es un listado de competencias agrupadas en 4 categorías: a) práctica profesional; b) proceso analítico; c) productos técnicos, y d) comunicaciones interpersonales. El comité define cada competencia en términos que facilite su comprensión y aplicación para el diseño curricular. Con ello, el CAC finaliza su labor.

En la etapa 5 se diseñan -o rediseñan cuando ya existenlos módulos a partir de las competencias identificadas. La clave de este proceso reside en alinear cuidadosamente los objetivos de cada módulo y las competencias que deben lograrse en ellos. Esto requiere trasladar los objetivos y metas existentes para cada módulo en aquellas competencias que se encuentran en la lista desarrollada en la etapa 4 y desarrollar las actividades de aprendizaje a realizarse dentro del módulo para que los estudiantes adquieran las competencias pertinentes y las actividades de evaluación conducentes a determinar si las competencias han sido logradas.

En la etapa 6 se ejecuta un programa que evalúe la efectividad del currículo para que los estudiantes logren las competencias establecidas.

Obsérvese que bajo este modelo el acento está puesto en la identificación de las competencias y en la evaluación del plan de estudios, pero no especifica método alguno para identificar los módulos que debe incluir el currículo ni su contribución a la formación de las competencias identificadas.

El otro modelo, llamado TTTI - St. Xavier Model ha sido desarrollado en el Technical Teachers Training Institute e implementado en el St. Xavier's Technical Institute, Mumbai, India, para el diseño curricular en el campo de la ingeniería [9].

Bajo este modelo se asume que el punto de partida para el desarrollo de cualquier currículo está dado por el perfil del profesional que se desea formar y que suele expresarse en un reducido número de objetivos generales que se aspira alcanzar, entendiendo que los objetivos son sentencias abiertas que permiten guiar la construcción de un currículo. Por ejemplo, un objetivo puede ser "producir personal técnico para las empresas a un nivel de supervisión". 
Las metas, en contraste con los objetivos, son sentencias más específicas que se derivan de los objetivos con miras a la estructuración de los módulos o cursos. Ellas indican los resultados esperados de un programa educacional. Generalmente, se tienen más metas que objetivos por cuanto un objetivo suele asociarse a más de una meta. Por ejemplo, una meta puede ser "producir ingenieros que trabajen como empresarios y/o supervisen el trabajo en los sectores informático y de servicios".

Definidas las metas se especifican las competencias requeridas para alcanzarlas, para posteriormente desagregarse en subcompetencias o capacidades. Finalmente, para el logro de estas capacidades subdivididas en cognitivas, procedimentales o prácticas y actitudinales se precisan los contenidos o materias a abordar.

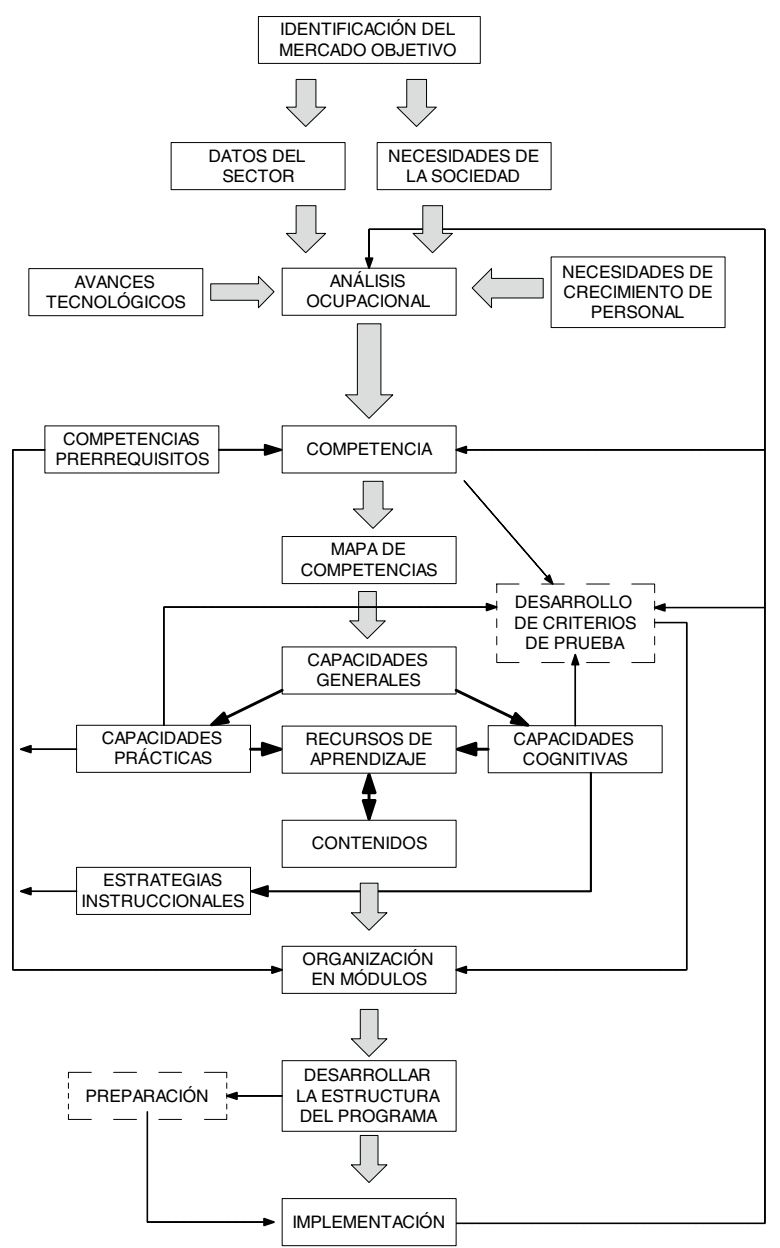

Figura 4. Modelo TTTI -St. Xavier. Fuente: [9].
En la figura 4 se presenta el modelo en el que el tanto el perfil profesional con sus objetivos y metas se desprenden del análisis ocupacional a realizar. Obsérvese que el énfasis está centrado en la identificación de las necesidades y las competencias asociadas a ellas, así como en la estructuración u organización de los módulos, pero no aborda un tema crucial: ¿cuál es el método de conversión de competencias en los módulos responsables de que los estudiantes tengan las competencias identificadas? ¿Cuáles son los módulos que deben configurar el currículo?

\section{MODELO CONCEPTUAL}

En los modelos analizados no se observa un método formal que guíe el proceso de identificación, secuenciación, estructuración y diseño de los módulos que configurarán el currículo. De allí que este trabajo se centre en esta área.

De lo visto se desprende que toda escuela responsable de la formación de profesionales por competencias busca integrar el contexto educativo con el laboral y social. Se asume que dentro de una universidad la unidad organizacional responsable de la formación de un profesional en particular es la Escuela.

El modelo que se propone debe ser capaz de sustentar un sistema de información que registre los datos necesarios para el cálculo de los indicadores y la realización de los análisis y seguimientos de los procesos que tienen lugar con miras a efectuar las evaluaciones pertinentes y realizar en el tiempo las modificaciones en el currículo que de ellas se desprendan. Modificaciones que pueden emanar de cambios en las competencias exigidas por el mercado laboral, en las características del alumno que ingresa a la universidad, en las prácticas pedagógicas, en los docentes y/o las tecnologías de información y comunicación disponibles.

El modelo conceptual de información subyacente en la metodología que se propone es el que se muestra en la figura 5. 


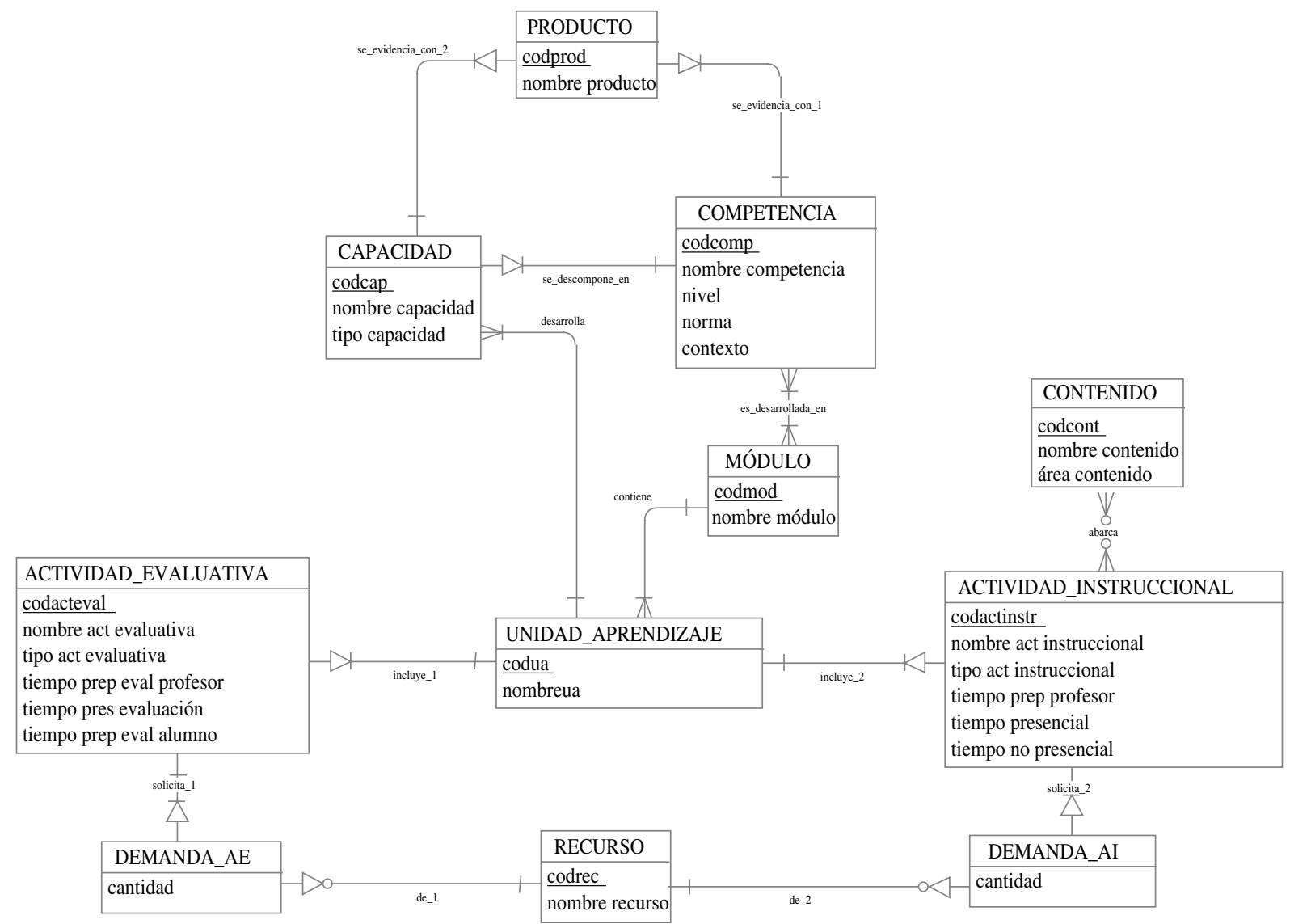

Figura 5. Modelo conceptual.

En él se asume que:

$\checkmark$ El logro de una competencia y/o capacidad se evidencia a través de uno o más productos (informes, experiencias, presentaciones, demostraciones, resultados, etc.);

$\checkmark$ Toda competencia requiere la articulación y puesta en acción de un conjunto de capacidades y, a su vez, toda capacidad puede ser requerida en más de una competencia;

$\checkmark$ Todo módulo desarrolla al menos una competencia, en tanto que toda competencia puede ser desarrollada en más de un módulo;

$\checkmark \quad$ Un módulo está conformado por al menos una unidad de aprendizaje, cada una de las cuales está contenida en un único módulo;

$\checkmark$ Toda unidad de aprendizaje es responsable del de una o más capacidades en los alumnos, incluyendo al menos una actividad evaluativa y al menos una actividad instruccional. Esta última abarca uno o más contenidos y cada contenido puede encontrarse en más de una actividad instruccional;
Tanto las actividades evaluativas como instruccionales demandan distintas cantidades de recursos de todo tipo.

\section{METODOLOGÍA PROPUESTA}

En el caso de la Universidad de Talca, para identificar los perfiles profesionales a formar se ha empleado la metodología DACUM (Developing A CurriculUM) creado en Canadá y desarrollado en los Estados Unidos, en la Universidad de Ohio, en la década de los años sesenta. Esta metodología - de carácter participativo que opera bajo una modalidad de taller- ${ }^{4}$ fue concebida como un instrumento para analizar ocupaciones y procesos de trabajo, generando insumos para conducir procesos de análisis funcional y poner en práctica una relación más estrecha entre la institución formadora de competencias y las empresas. En la medida que DACUM analiza el proceso productivo y las tareas que se derivan de ello, se construye un currículo basado en el trabajo real [13].

\footnotetext{
4 Por lo que la metodología también recibe el nombre de taller DACUM.
} 
En la figura 6 se presenta una propuesta metodológica de diseño curricular centrada en la conformación del currículo, plan de estudios o malla curricular a partir de un perfil profesional dado por las competencias y capacidades asociadas a él, que resultan del taller DACUM.

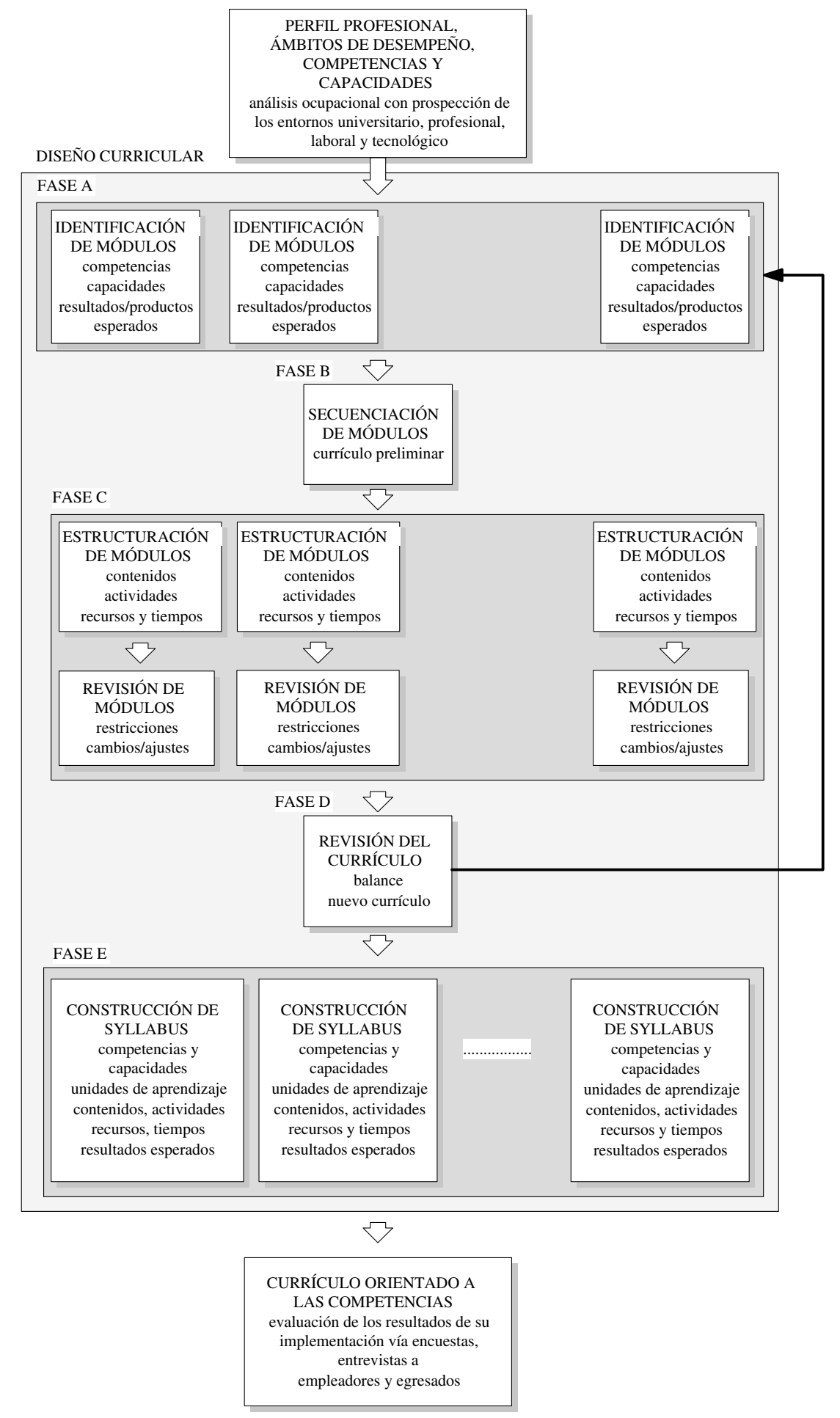

Figura 6. Metodología propuesta. 
El contenido de cada fase dentro de la metodología propuesta se expone a continuación.

\section{Fase A: Identificación de módulos a partir de las competencias}

Se considera como punto de partida el vector de competencias identificadas en el mercado laboral (de salida) que se aspira satisfacer, al que se asocia un vector inicial de módulos equivalente. Esto es, a cada competencia le está asociado un módulo responsable de la competencia correspondiente. En un principio el nombre del módulo asume el nombre de la competencia de la que se hace cargo. Este nombre puede modificarse para facilitar su reconocimiento y la comunicación con el entorno académico-profesional.

En esta fase el mercado laboral (de salida) tiene un rol protagónico en la sentencia asociada a la competencia, el producto esperado y las capacidades requeridas para el logro de la competencia, en tanto que los contenidos del módulo son de responsabilidad de los profesores. La sentencia referida a la competencia que se debe alcanzar al finalizar el módulo está dada por las exigencias del sector en la que se desempeñará el profesional, debe contener el nivel de desempeño esperado y el contexto en que se espera dicho nivel de desempeño.

Obsérvese que la información resultante-competencia que se debe alcanzar, resultados o productos esperados al finalizar el módulo y capacidades que se requieren para el logro de la competencia- es de especial interés para los tomadores de decisiones, los profesores, los estudiantes, y los evaluadores.

\section{Fase B: Secuenciación de los módulos}

Identificados los módulos corresponde secuenciarlos en el tiempo en base a un criterio de precedencia o relación de orden siguiendo alguno de los modelos de aprendizaje (de lo teórico a lo práctico, de la práctica a lo conceptual, de lo simple a lo complejo, de lo concreto a lo abstracto o viceversa, etc.). Se asume que todo módulo puede no tener, o tener uno a más módulos prerrequisitos o correquisitos (modulo(s) que deben ser realizados en forma simultánea). Como resultado debe tenerse una primera malla o plan de estudios expresado gráficamente.

\section{Fase C: Estructuración de los módulos identificados}

Teniendo a la vista una primera aproximación del plan de estudios se debe proceder a estructurar cada uno de los módulos. Esto implica que la información de salida de esta fase debe incluir los contenidos que se abordarán y una estimación de los tiempos y recursos comprometidos (bibliografía básica, infraestructura, entre otros).

\section{Fase D: Revisión de módulos y/o competencias}

Una vez conocidas las competencias, los contenidos, los recursos y tiempos comprometidos en cada uno de los módulos, el tiempo total que las actividades de aprendizaje demanden del alumno (incluye tiempos de aula, laboratorio o trabajo de campo, de trabajo y/o lectura personal, desarrollo de casos, etc.) deberá enmarcarse dentro de las restricciones vigentes en la organización. A modo de ejemplo, que el tiempo total de cada módulo esté dentro del régimen curricular de la Universidad (semestral, trimestral, anual, etc). Normalmente estos tiempos inicialmente expresados en horas semanales suelen transformarse en créditos equivalentes ECTS $^{5}$.

De igual modo puede darse que la suma de los tiempos que los alumnos deben destinar a los módulos excede el tiempo total asignado a la formación y que viene dado por la duración del plan de estudios.

Estas restricciones pueden conducir a la necesidad de revisar el módulo y/o la competencia. En caso que el módulo resultara ser "muy grande", puede ser una señal o invitación a revisar la competencia y/o el módulo, ya sea por la vía de redefinir la competencia en más de una competencia, o bien, particionar el módulo comprometido. En el otro caso, si el módulo resultara ser "muy pequeño", es posible que la competencia pueda acoplarse a otra competencia ya identificada.

Por tanto, la correspondencia biunívoca entre competencia y módulo puede verse alterada como resultado del análisis de tiempos posibilitando la existencia de módulos que desarrollen más de una competencia, así como de competencias que sean desarrolladas en más de un módulo. Sin embargo, dentro de lo posible se debe tratar de preservar la relación uno a uno entre módulo y competencia. No obstante el Modelo Conceptual propuesto contempla la posibilidad que esta correspondencia no sea biunívoca, sino que múltiple.

\section{Fase E: Revisión del plan de estudios}

El plan de estudios preliminar construido consideró exclusivamente las competencias prerrequisitos y/o correquisitos para cursar un nuevo módulo. Sin embargo, no

\footnotetext{
5 En la Universidad de Talca se está utilizando el concepto ECTS proveniente del ámbito europeo, donde el total de créditos ECTS corresponde al total de horas presenciales y de trabajo autónomo que se estima todo alumno debe destinar al módulo pertinente para cursarlo satisfactoriamente dividido por 27.
} 
toma en consideración el necesario balance de los tiempos en cada período académico. Es en esta etapa que debe procederse a revisar el plan de estudios construido, lo que pudiera motivar desplazamientos de módulos, cambios en los prerrequisitos y/o correquisitos que obliguen a replantear las competencias y módulos ya estructurados, forzando a volver a la Fase A.

Incluso puede darse el caso que se tenga que objetar la viabilidad del logro de las competencias consignadas en el perfil profesional definido, obligando a modificarlo en función de las limitaciones o restricciones dadas por el régimen de estudios de la institución.

\section{Fase F: Construcción de los syllabus}

A partir de las capacidades y contenidos que se aspira lograr en cada módulo, se debe estar en condiciones de elaborar el syllabus correspondiente. En consecuencia, deberán construirse tantos syllabus como módulos se hayan estructurado. En la Universidad de Talca el syllabus es el documento oficial que contiene la organización de las unidades de aprendizaje, sus contenidos, recursos involucrados y los tiempos que demanden las actividades instruccionales y evaluativas -tanto a estudiantes como a profesores- junto con los niveles de logro y desempeño, dando cuenta de la secuenciación, coherencia y progresión entre las competencias y capacidades vinculadas a las unidades de aprendizaje.

Para el caso de carreras profesionales ya existentes, se observa que bajo la metodología propuesta no se considera el plan de estudios vigente, asumiéndose que se parte con "una hoja en blanco". Esta concepción tiene por fin dejar de lado "historias" que muchas veces tienen más que ver con influencias, intereses, personalidades o grupos de poder académico. Esta presencia en los planes de estudio puede ser contraproducente en la confección de un currículo orientado a las competencias. Sin perjuicio de lo expuesto, nada impide que el plan de estudios resultante de la metodología propuesta sea contrastado con el vigente y experimentar una nueva revisión si las circunstancias lo aconsejan.

\section{DISCUSIÓN}

Tradicionalmente el currículo se ha construido sobre una base disciplinar y centrado en la adquisición por parte de los alumnos de capacidades cognitivas. En la Universidad de Talca se ha optado por un diseño curricular basado en competencias en el que importen no sólo las capacidades cognitivas (saber conocer), sino las procedimentales (saber hacer) y actitudinales personales e interpersonales (saber ser y estar). Por ello el agrupamiento (o aglomeración) para concebir las asignaturas o módulos deja de centrarse sólo en contenidos de una misma disciplina.

Con el foco puesto en las competencias que deben tener los estudiantes al momento de egresar, el agrupamiento viene a estar dado por las capacidades que demanda cada competencia. De allí que bajo la metodología propuesta se parte asociando un módulo a una competencia. En las etapas siguientes se especifican las distintas capacidades que se deben formar para el logro cada competencia, así como los productos, las actividades instruccionales y evaluativas a desarrollar, los tiempos involucrados y la secuencia que debe establecerse entre los módulos.

Si bien las etapas son secuenciales, en todo momento se debe estar disponible para revisar lo realizado y efectuar las modificaciones que sean pertinentes. Por tanto, la linealidad de las etapas está sujeta a la posibilidad de "volver atrás", aun cuando conlleve modificaciones sustanciales.

Esto importa por razones prácticas, a fin de "ajustar" el currículo a las restricciones imperantes, las que pueden ir de limitaciones institucionales - por ejemplo, que en todo período académico el conjunto de módulos en un currículo no pueda exceder un total de créditos u horas por sobre una cantidad dada-; limitaciones de recursos de aprendizaje -laboratorios, computadores, materiales, herramientas, etc.-, o bien, limitaciones de secuenciación considerando que la duración de un currículo no pueda exceder un total de períodos académicos (años, semestres o trimestres). Lo anterior puede llevar al extremo de replantear el vector de competencias del perfil profesional asociado. Una vez diseñado y puesto en acción el currículo, todo cambio en el perfil profesional como consecuencia del dinamismo del mercado laboral debiera afectar el currículo vigente. Tomando en cuenta la correspondencia entre módulo y competencia, su modificación se ve facilitada con la metodología propuesta. Las situaciones que se pueden presentar son las siguientes:

a) si al perfil profesional se incorpora una nueva competencia, su consecuencia debe ser incorporar un nuevo módulo en el currículo o insertarla dentro de un módulo existente luego de una revisión de él;

b) si al perfil profesional se elimina una competencia, su consecuencia debe ser eliminar y/o modificar el(los) módulo(s) involucrados;

c) si al perfil profesional se modifica una competencia (incorporan nuevas capacidades, o eliminan/modifican algunas de ellas), su consecuencia debe ser modificar el(los) módulo(s) afectado(s).

Bajo la metodología propuesta, tales modificaciones se ven facilitadas dado que permite "aislar" las competencias 
afectadas. En consecuencia, la metodología no sólo nos proporciona un curso de acción para diseñar un currículo, sino que para mantener un currículo orientado a las competencias. Esto último es de particular relevancia cuando se está en un entorno tan volátil como el actual.

\section{CONCLUSIONES}

La metodología planteada aborda un tema no resuelto a la fecha: el de la "conversión" de un vector de competencias en un currículo o plan de estudios mediante un conjunto de etapas que se ciñen a una lógica.

La propuesta metodológica se está implementando a nivel de una carrera en particular -Ingeniería en Informática Empresarial- en la Universidad de Talca, y dada la carencia de soporte metodológico existente en la materia, ella ha permitido construir un currículo orientado a la formación de competencias sin grandes dificultades en la medida que exista un perfil profesional traducido en un vector de competencias delimitadas. Las mayores dificultades residen en la confección de los módulos y la interrelación entre ellos, particularmente cuando no existe claridad respecto de las capacidades que demandan las competencias.

A futuro, esta misma propuesta será sometida a prueba para los efectos de las modificaciones en el currículo que la cambiante realidad científico-tecnológica y laboral aconseje.

\section{AGRADECIMIENTOS}

Este trabajo ha sido desarrollado en el marco del proyecto MECESUP de Rediseño Curricular de la Universidad de Talca (TAL0101) con que se ha apoyado a la Escuela de Informática Empresarial.

\section{REFERENCIAS}

[1] MINEDUC. "Una Mirada a la Educación: Resultados del Proyecto Mundial de Indicadores WEI 2003”. Ministerio de Educación, Nota Técnica. Septiembre 2003. Fecha de consulta: 2 de marzo de 2005. URLs: www.mineduc.cl

[2] N. Sutcliffe, S.S Chan and M. Nakayama. "A Competency Based MSIS Currículo”. Journal of Information Systems Education. Vol. $16 \mathrm{~N}^{\mathrm{o}} 3$, pp. 301-309. Fall 2005.
[3] D. McClelland. "Testing for Competence rather than for Intelligence". 1973.

[4] A.M. Larraín y L.E. González. "Formación Universitaria por Competencias". Seminario Internacional CINDA. Currículo Universitario basado en Competencias, p. 44. Universidad del Norte. Barranquilla, Colombia. 25-26 julio 2005.

[5] R. Posada. "Formación Superior basada en competencias, interdisciplinariedad y trabajo autónomo del estudiante". Revista Iberoamericana de Educación. ISSN: 1681-5653. Facultad de Educación. Universidad del Atlántico. Colombia. Fecha de consulta: 24 de marzo de 2007. URLs: www.campus-oei.org/revista/edu_sup22.htm

[6] IBSTPI. Competencies, International Board of Standards for Training and Perfomance Instruction, 2005. URLs: http://www.ibstpi.org/ competencies. htm

[7] NCES. "Defining and assessing learning: Exploring competency-based iniciatives". Nacional Center for Education Statistics. 2002. Fecha de consulta: 15 de marzo de 2007. URLs: nces.ed.gov/ pubs2002/2002159.pdf

[8] M. Sladogna. "Una mirada a la construcción de las competencias desde el sistema educativo. La experiencia Argentina”. CINTERFOROIT. Competencias laborales en la formación profesional. Boletín Técnico Interamericano de Formación Profesional $\mathrm{N}^{\circ} 149$, p. 15. Mayo-agosto 2000.

[9] J. Earnest y F.E. de Melo. "Competency-Based Engineering Curricula -an innovative approach". International Conference on Engineering Education. August 6-10, 2001. Oslo-Norway. Fecha de consulta: 20 de marzo de 2006. URLs: www.ineer. org/ Events/ICEE2001/Proceedings/papers/439. pdf

[10] B.S. Bloom. "Taxonomía de los objetivos de la educación”. Ed. El Ateneo. Buenos Aires, Argentina. $7^{\mathrm{a}}$ edición, pp. 355. 1979.

[11] R.H. Dave. "Taxonomy of Educational Objectives and Acievement Testing: Developments in Educational Testing". Proceedings of the International Conference of Educational Measurement. Vol. 2. University of London Press. Londres, Inglaterra. 1966. 
[12] S.Y. Chyung, D. Stepich y D. Cox. "Building a Competency-Based Currículo Architectura to Educate 21st-Century Business Practicioners". Journal of Education for Business. Vol. $81 \mathrm{~N}^{\circ} 6$, pp. 307-311. July-august, 2006.
[13] L. Mertens. DACUM y sus variantes SCID y AMOD. CINTERFOR-OIT. November 1997. 